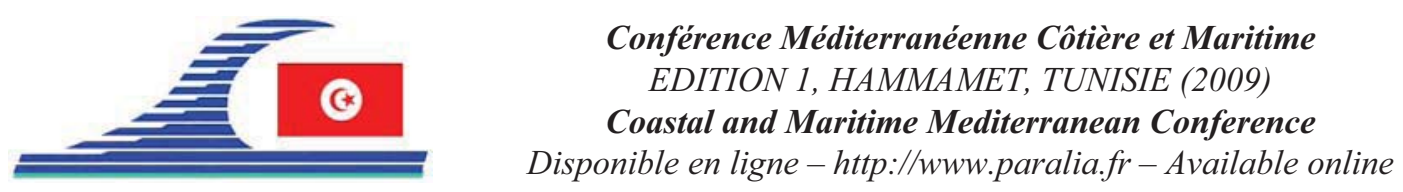

\title{
Etude de formulations d'un béton de sable à base de sable de dragage
}

\author{
Abdeljalil ZRI ${ }^{1,2}$, Nor Edine ABRIAK ${ }^{1,2}$, Mahfoud BENZERZOUR ${ }^{1,2}$ \\ 1. Université Lille Nord de France, F-59000 Lille, France. \\ 2. EMDouai, PC2A-CE, IA, MPE-TPCIM, MPE-GCE, F-59500 Douai, France. \\ zri@ensm-douai.fr
}

\section{Résumé :}

Dans un contexte de développement durable, la valorisation de sable de dragage dans le génie civil permet de faire face au manque de gisements et à l'épuisement de granulats. De cela émerge l'idée de réaliser à partir de ce déchet un nouveau matériau de construction. Il s'agit d'un béton de sable normalisé qui présente des caractéristiques mécaniques semblables aux bétons classiques. Ce matériau va permettre de recentrer économiquement la consommation de granulats alluvionnaires en exploitant les stocks de sable de dragage abondants. De ce point de vue, notre intérêt est, non pas de remplacer les bétons traditionnels par ce matériau, mais de proposer une alternative à ces bétons pour certaines applications.

La carte d'identité établie pour l'ensemble des constituants entrant dans la formulation suite à une caractérisation physico-chimique révèle que ce matériau de base est classé comme déchet inerte. En outre, le sable de dragage est dépourvu de fines $(<1 \%)$. A cet effet, l'incorporation des additions est justifiée afin de combler les vides et d'apporter un pouvoir pouzzolanique supplémentaire à la formulation.

A l'issue de ce travail, on aboutit à la formulation d'un matériau normalisé pour la classe environnementale humide avec gel modéré et qui se caractérise par des résistances mécaniques très performantes supérieures à celles des bétons classiques.

\section{Mots clés :}

Béton de sable - Sable de dragage - Valorisation - Maniabilité - Compacité

\section{Introduction}

En France, le domaine de la construction consomme plus de 365 millions de tonnes de granulats par an. D'un autre côté, le volume annuel de sédiments dragués est estimé à $50 \mathrm{Mm}^{3}$ (ALZIEU \& QUINIOU, 1999). Par conséquent, ce volume énorme de matériau peut constituer une source d'approvisionnement en granulats pour un secteur consommateur tel que le génie civil. Le domaine du bâtiment consomme $22.4 \%$ de granulats contre $50.7 \%$ pour le domaine routier (MICHEL, 1997). La présente étude vise à valoriser ce matériau marin pour établir un béton de sable normalisé à base de sable de dragage. Notre travail consiste à choisir les constituants adéquats (fillers, DOI: $10.5150 / \mathrm{cmcm} .2009 .017$ 
superplastifiant...) et à définir leurs proportions dans le mélange afin d'obtenir un matériau de construction à l'instar du béton traditionnel, qui répond aux prescriptions de la normalisation pour la classe environnementale humide avec gel modéré ( $2 \mathrm{~b} 1$ : selon la NF P 18-305 ou XF1: selon la norme NF EN 206-1) : cas général dans le département du Nord de la France. La particularité de béton de sable formulé et de celui du projet SABLOCRETE (SABLOCRETE, 1994) est que son matériau de base est considéré comme un déchet (décret n 2002-540 du 18 avril 2002, rubriques 170505 et $170506)\left(\mathrm{JO} \mathrm{n}^{\circ} 93 \mathrm{du} 20\right.$ avril 2002) avec un $\mathrm{D}_{\max }$ inférieur à $1 \mathrm{~mm}$ et dépourvu des fines $(<1 \%)$. Par conséquent, l'incorporation du fillers est indispensable pour combler les vides entre les grains de sable. La question qui se pose est de savoir quel est l'ajout le plus adéquat en termes de résistance et d'économie. La finalité principale de ce travail est de trouver le mélange optimal possible entre le sable de dragage et un ou plusieurs ajouts avec du ciment et de l'eau afin d'obtenir des caractéristiques mécaniques satisfaisantes. Le sable de dragage étudié est d'origine marine; il s'agit du sable déposé à la Capitainerie Ouest du Port Autonome de Dunkerque.

La démarche adoptée débute par une caractérisation complète afin de déterminer la carte d'identité pour chaque constituant entrant dans la formulation à savoir (sable de dragage, ciment, laitier, cendres volantes et fumée de silice) (ZRI et al., 2009). Puis, on procède à la formulation en tenant en compte des contraintes exigées par les normes en vigueur. La méthode de formulation obtenue sera applicable pour les trois additions choisies (laitier de haut fourneau, fumée de silice et cendres volantes). Enfin, pour répondre aux objectifs fixés, on effectue des mesures à l'état frais et durci sur chaque formulation.

\section{Méthode de formulation}

L'étude de formulation consiste à trouver le mélange optimal qui répond aux critères techniques et économiques fixés dans la norme. Compte tenu de la granularité de ce matériau, les méthodes utilisées pour la formulation du béton usuel qui consistent généralement à définir une courbe granulaire de référence ne sont pas applicables. Par conséquent, la formule de Caquot est adoptée car elle donne la porosité minimale théorique du béton à partir de l'étendue granulaire du matériau. D'ailleurs, cette formule est la base des méthodes de formulation des bétons de Bolomey, Faury et Dreux. Selon Caquot, la porosité minimale se décompose en la somme d'un volume d'eau et d'un volume piégé, selon la formule suivante :

$$
(\mathrm{e}+\mathrm{v}) \min =0.8(\mathrm{~d} / \mathrm{D})^{0,2}
$$

où : $d / D$ est l'étendue granulaire $y$ compris les fines.

\section{Formulation du béton de sable à base de sable de dragage}

La porosité obtenue par la méthode présentée est similaire à celle donnée par le logiciel René-LCPC pour un indice de compaction de 4.75. Le recours à ce logiciel est adopté, 
d'un côté pour confirmer les résultats obtenus; d'un autre côté pour minimiser le nombre de gâchées. L'ensemble des formulations de béton de sable à base de sable de dragage et les additions effectuées est présenté dans le tableau 1. Les caractéristiques des bétons de sable à l'état frais et durci sont présentées dans le tableau 2.

Tableau 1. Formulation des bétons de sable à base de sable de dragage.

\begin{tabular}{lcccccccc}
\hline \multicolumn{1}{c}{ Béton } & $\mathrm{F}_{1}$ & $\mathrm{~F}_{2}$ & $\mathrm{~F}_{3}$ & $\mathrm{~F}_{4}$ & $\mathrm{~F}_{5}$ & $\mathrm{~F}_{6}$ & $\mathrm{~F}_{7}$ & $\mathrm{~F}_{8}$ \\
\hline Ciment $\left(\mathrm{kg} / \mathrm{m}^{3}\right)$ & 362.5 & 340 & 314.4 & 331.2 & 349.5 & 436.3 & 351 & 320.8 \\
$\mathrm{SD}\left(\mathrm{kg} / \mathrm{m}^{3}\right)$ & 1563.6 & 1360 & 1342.8 & 1669.5 & 1479.7 & 1354.3 & 1303 & 1485.1 \\
$\mathrm{E}\left(\mathrm{l} / \mathrm{m}^{3}\right)$ & 256.8 & 249 & 214.3 & 237.2 & 245.2 & 233.5 & 272 & 229.2 \\
Laitier $\left(\mathrm{kg} / \mathrm{m}^{3}\right)$ & 135.4 & 145 & -- & -- & 115.3 & -- & -- & 137 \\
Fumée de silice $\left(\mathrm{kg} / \mathrm{m}^{3}\right)$ & -- & -- & -- & 33.1 & -- & -- & 147 & -- \\
Cendres volantes $\left(\mathrm{kg} / \mathrm{m}^{3}\right)$ & -- & -- & 152.4 & -- & -- & 186.7 & -- & -- \\
SP $\left(1 / \mathrm{m}^{3}\right)$ & 10 & 10 & 16 & 10 & 10 & 10 & 10 & 10 \\
E/L & 0.54 & 0.54 & 0.56 & 0.61 & 0.56 & 0.40 & 0.56 & 0.53 \\
$\mathrm{~A} /(\mathrm{C}+\mathrm{A})$ & 0.27 & 0.3 & 0.33 & 0.09 & 0.25 & 0.3 & 0.3 & 0.3 \\
E/C & 0.73 & 0.75 & 0.72 & 0.74 & 0.72 & 0.55 & 0.79 & 0.74 \\
$\%$ SP & 2 & 2 & 3 & 3 & 2 & 2 & 2 & 2 \\
$\%$ extrait sec & 1 & 1 & 1 & 1 & 1 & 0 & 1 & 1 \\
\hline
\end{tabular}

SD: Sable de dragage. E: eau. SP: Superplastifiant. C: Ciment. A: Fillers. L: Liant équivalent $(C+k A)$

Tableau 2. Caractéristiques des bétons frais et propriétés mécaniques des bétons durcis.

\begin{tabular}{ccccccccc}
\hline Béton & $\mathrm{F}_{1}$ & $\mathrm{~F}_{2}$ & $\mathrm{~F}_{3}$ & $\mathrm{~F}_{4}$ & $\mathrm{~F}_{5}$ & $\mathrm{~F}_{6}$ & $\mathrm{~F}_{7}$ & $\mathrm{~F}_{8}$ \\
\hline Maniabilité & 17 & 8 & 20.78 & $\mathrm{Ferme}$ & 11 & 6 & 7 & 19 \\
Rt7 $(\mathrm{MPa})$ & 5.61 & 5.93 & 3.97 & 3.74 & 6.35 & 6.08 & 6.23 & 7.56 \\
Rt14 $(\mathrm{MPa})$ & 3.73 & 6.48 & 3.44 & 1.97 & 6.56 & 7.14 & 3.7 & 9.19 \\
Rt28 $(\mathrm{MPa})$ & 7.22 & 5.04 & 2.98 & 2.11 & 7.43 & 7.26 & 6.22 & perdu \\
Rc7 $(\mathrm{MPa})$ & 32.56 & 31.65 & 31.23 & 19.60 & 28.98 & 40.30 & 38.13 & 44.25 \\
$\mathrm{Rc14}(\mathrm{MPa})$ & 39.37 & 38.16 & 22.32 & 24.12 & 32.81 & 45.67 & 44.9 & 52.76 \\
$\mathrm{Rc} 28(\mathrm{MPa})$ & 45.83 & 46.06 & 24.33 & 26.11 & 37.94 & 50.81 & 55.33 & 66.66 \\
\hline & Rc : résistance à la compression ; Rt : résistance à la traction & &
\end{tabular}

\section{Conclusion}

L'étude menée montre la faisabilité d'utilisation de sable de dragage dans le béton. Autrement dit la substitution des gros granulats du béton classique par ce matériau est envisageable pour certaines applications du domaine de la construction. La méthode de formulation adoptée répond parfaitement aux attentes souhaitées car elle permet de respecter les rapports exigés par la norme en termes de dosage entre l'ajout et le ciment 
ainsi que le rapport E/L fixé préalablement. Les résultats obtenus ont montré, d'une part, que le béton de sable à base de sable de dragage présente des résistances mécaniques performantes avec une maniabilité fluide à plastique et d'autre part, que le coefficient d'activité du laitier broyé est élevé par rapport aux autres ajouts. L'incorporation des ajouts dans les formulations a permis de réduire la quantité du ciment dans les mélanges en comparaison aux bétons traditionnels avec des résistances mécaniques très satisfaisantes. Une étude numérique et paramétrique est en cours d'élaboration. Cette étude permettra l'optimisation de formulations et de tester leurs performances à plus grande échelle.

\section{Références}

ALZIEU C., QUINIOU F. (1999). Dragages et environnement marin. Etats des connaissances-comportement des polluants. Institut français de recherche pour l'exploitation de la mer, $223 \mathrm{p}$.

MICHEL F. (1997). Les granulats. Union nationale des producteurs de granulats, 40 p. SABLOCRETE (1994). Bétons de sable: caractéristiques et pratiques d'utilisation. Presses de l'Ecole Nationale des Ponts et Chaussées, 237 p.

ZRI A., ABRIAK N.E., ZENTAR R. (2009). Etude comparative de comportement physico-mécanique d'un sédiment pollué et non pollué. Revue Déchets Sciences et Techniques, ${ }^{\circ}$ 53, pp 13-19. 